\title{
Detection of Stress Hormone in the Milk for Animal Welfare Using QCM Method
}

\author{
Takeshi Ito, ${ }^{1}$ Nobuyoshi Aoki, ${ }^{2}$ Akihisa Tsuchiya, ${ }^{2}$ Satoru Kaneko, ${ }^{2}$ \\ Kiyoshi Akiyama, ${ }^{3}$ Katsuji Uetake, ${ }^{4}$ and Koji Suzuki ${ }^{5}$ \\ ${ }^{1}$ Faculty of Engineering Science, Kansai University, Yamate-cho 3-3-35, Suita, Osaka 564-8680, Japan \\ ${ }^{2}$ Kanagawa Institute of Industrial Science and Technology, Shimo-imaizumi 705-1, Ebina, Kanagawa 243-0435, Japan \\ ${ }^{3}$ Livestock Industry Technology Station, Hongo 3750, Ebina, Kanagawa 243-0417, Japan \\ ${ }^{4}$ School of Veterinary Medicine, Azabu University, Fuchinobe 1-17-71, Chuo, Sagamihara 252-5201, Japan \\ ${ }^{5}$ Faculty of Science and Technology, Keio University, Hiyoshi 3-14-1, Kohoku, Yokohama 223-8522, Japan
}

Correspondence should be addressed to Takeshi Ito; t.ito@kansai-u.ac.jp

Received 16 May 2017; Accepted 10 July 2017; Published 17 September 2017

Academic Editor: Takemi Matsui

Copyright (C) 2017 Takeshi Ito et al. This is an open access article distributed under the Creative Commons Attribution License, which permits unrestricted use, distribution, and reproduction in any medium, provided the original work is properly cited.

\begin{abstract}
We developed a rapid and sequential analysis system to determine stress marker in the milk. One of the famous stress markers, cortisol, was detected using our method. Quartz crystal microbalance (QCM) method with a twin sensor was used in this study. One channel detected stress marker corresponding to antigen-antibody interaction and the other channel was used as a reference to remove environmental influences. Although nonspecific adsorption was monitored on each channel, frequency difference between them was within a few $\mathrm{Hz}$ on the injection of sample solution. One determination cycle including regeneration step could be performed within 10 minutes. The system could detect the cortisol level from $0.1 \mathrm{pg} / \mathrm{mL}$ to $100 \mathrm{pg} / \mathrm{mL}$. These results show that our system has a potential to check the daily feeding condition for cows in terms of animal welfare.
\end{abstract}

\section{Introduction}

Many biomarkers exist in biological fluid, for example, blood, urine, saliva, and milk. To assess daily health condition, noninvasive monitoring method of biomarker is desired for not only human but also animals. Animals including human face many stressors by life environments and physiological problems. Long-term and strong stress might cause not only mental diseases such as depression but also heart disease and high blood pressure for human. Animals also feel stress; for example, stress hormone level of abandonment dogs in Fukushima was 5 to 10 times larger than that in other areas in Japan after the big earthquake of March 11, 2011 [1].

Animal welfare is recently focussed in the livestock industry [2-5]. Controlling the stress level of farm animals is important not only in ethical concerns but also in highquality production. Farm animals face many stressors around the feeding environment such as temperature, feed, and the number of animals per unit area. We focussed on a cow for an actual case. Cortisol is well known as a stress marker of the endocrine system. Some reports monitored cortisol level in cow's blood [6-9], and the concentration of cortisol was lower than $20 \mathrm{ng} / \mathrm{mL}$. However, collecting blood is stressful for animals and controller. Noninvasive monitoring of cortisol level is desired for the daily stress test. Milk is ideal material as an analyte, since the milk is collected in a daily operation. However, the concentration of cortisol in the milk is lower than that in the blood [10-15]; it ranged from about $500 \mathrm{pg} / \mathrm{mL}$ to $10 \mathrm{ng} / \mathrm{mL}$. In addition, many foreign substances are included in the milk. A detection system for cortisol requires high sensitivity with short detection time for livestock industry. We report primitive and positive data on stress monitoring for livestock industry.

Many methods were used for detecting cortisol in the milk. Radioimmunoassay (RIA) [10-12] and enzyme-linked immunosorbent assay (ELISA) [13-15] are well known as highly sensitive detection methods of cortisol. However, these techniques require time-consuming steps for secondary 
TABLE 1: Surface treatment conditions and their properties of contact angle and the delta meaning frequency differences between Ch1 and Ch2 on the injection of 10 vol\%-plain-milk-containing sample.

\begin{tabular}{|c|c|c|c|c|}
\hline & 1st reagent & 2 nd reagent & Contact angle $\left[{ }^{\circ}\right]$ & Delta $[\mathrm{Hz}]$ \\
\hline (a) & $\mathrm{N} 102,1 / 3$ & Not used & 55 & -44 \\
\hline (b) & IAS & Not used & 61.4 & 36 \\
\hline (c) & Casein, 0.25 wt $\%$ & Not used & 58.2 & -10 \\
\hline (d) & Plain milk, 1/10 & Not used & 45 & -20 \\
\hline (e) & $\mathrm{N} 102,1 / 3$ & Plain milk, 1/10 & 61.1 & -2 \\
\hline (f) & $\mathrm{N} 102,1 / 3$ & Skimmed milk & 57.3 & -2.2 \\
\hline (g) & $\mathrm{N} 102,1 / 3$ & Casein, $0.25 \mathrm{wt} \%$ & 58.8 & -4 \\
\hline
\end{tabular}

labeling and reactions. In addition, the handling of hazardous radioisotopes limits widespread use on RIA. We focused on quartz crystal microbalance (QCM) method, which is one of label-free and real-time measurement of antigen-antibody interaction with simplicity, convenience, and low cost [1620]. Our group reported that QCM method could detect cortisol level ranging from 5 to $100 \mathrm{pg} / \mathrm{mL}$ [16]. However, no study has been published for detecting cortisol level in the milk using the QCM method. The major problem of the QCM method is the slightly large noise level caused by measurement environment, such as temperature and density of surrounding media. Since twin sensor removes these environmental influences, noise level can be decreased drastically $[16,17]$. In this report, one channel was used as a reference (Ch1) and the other channel measured antigen-antibody interaction (Ch2). Difference of frequency shift between Ch2 and Ch1, delta, was corresponding to the amount of analyte bound on Ch2 without environmental influence. In addition, competitive assay was applied in this report, because the molecular weight of cortisol was too small to be detected directly on QCM. Sequential analysis system is desired for laboratory or cow-house use. Our sensing system is integrated with a flow injection analysis and the condition of regeneration was described in this paper. We reported primitive and positive data for stress monitoring using twin sensor QCM for the animal welfare.

\section{Materials and Methods}

2.1. Materials. Monoclonal antibody to cortisol (anti-Crt) was obtained from HyTest Ltd. (Turku, Finland). Cortisol 3-BSA (Crt-BSA) was purchased from Fitzgerald Industries International (MA, USA). Cortisol standard solution (500 $\mathrm{ng} / \mathrm{mL}$ ) was obtained from Cayman Chemical Company (MI, USA). Blocking reagent, N102, was from NOF Corporation (Tokyo, Japan). Immunoassay stabilizer (IAS) was from Advanced Biotechnologies Inc. (MD, USA). Phosphate-buffered saline (PBS, $\mathrm{pH}=7.4$ ), casein, sodium hydroxide, and glycine hydrochloride were obtained from Wako Pure Chemical Industries, Ltd. (Osaka, Japan). Plain milk including 2\% milk fat was produced in Hokkaido (Japan) and purchased from a supermarket. Skimmed milk was obtained from Megmilk Snow Brand Co., Ltd. (Tokyo, Japan).
2.2. Coating of the Sensor Surfaces. The surface of the Au electrodes was treated using a preparation jig as follows. $170 \mu \mathrm{g} / \mathrm{mL}$ of anti-Crt was coated on Ch2 for 10 minutes firstly. After rinsing Ch2 with PBS, seven conditions of surface treatment were applied for Ch1 and Ch2 in the same way as shown in Table 1 . The blocking reagents were prepared as below. N102 was diluted by $1 / 3$ by adding PBS. IAS was used as it was. Plain milk was diluted by $1 / 10$ by adding PBS. $1.1 \mathrm{mg}$ of skimmed milk was dissolved in PBS. After casein was dissolved in $10 \mathrm{mM} \mathrm{NaOH}$ solution, concentration of casein was adjusted to $0.25 \mathrm{wt} \%$ by adding PBS. First, reagents were dropped on each sensor surface for 25 minutes. After rinsing with PBS, second reagents were dropped for 5 minutes. Here, (a), (b), (c), and (d) did not use secondary reagents. Finally, PBS was dropped on each sensor for 10 minutes and the surface was rinsed by PBS.

2.3. Sensor System and Measurement. Cortisol was detected using a NAPiCOS QCM system consisting of a thermostatic chamber and a frequency counter (Nihon Dempa Kogyo Co., Ltd.). Figure 1 shows the schematic figure of the QCM system integrated with a flow injection system and the twin sensor chip (inset). A micro syringe pump (Model 100, KD Scientific, MA, USA) and a sample injector (injection volume: $20 \mu \mathrm{L}$, 7125 , Rheodyne, WA, USA) were connected to a micro flow cell (inner volume: $5 \mu \mathrm{L}$ ) in the QCM system through PEEK tubes. A $30 \mathrm{MHz}$ twin sensor chip (PSA-E-3002T, Nihon Dempa Kogyo Co., Ltd.) had two Au electrodes, and each surface area of them was $7.75 \mathrm{~mm}^{2}$. PBS was used as a carrier solution. The prepared sensor chip was connected with a micro flow cell, and the assembled cell was set in the thermostatic chamber and kept at $25^{\circ} \mathrm{C}$. Sample solution was injected and frequency shifts on each channel were observed. Then, delta measurement $($ delta $=\mathrm{Ch} 2-\mathrm{Ch} 1)$ was performed at the flow rate of $6 \mu \mathrm{L} / \mathrm{min}$.

\section{Results and Discussion}

3.1. Sensing System. The basic principle of QCM sensor depends on the frequency shift by analyte adsorbed on the QCM resonator. The amount of the analyte adsorbed on the 


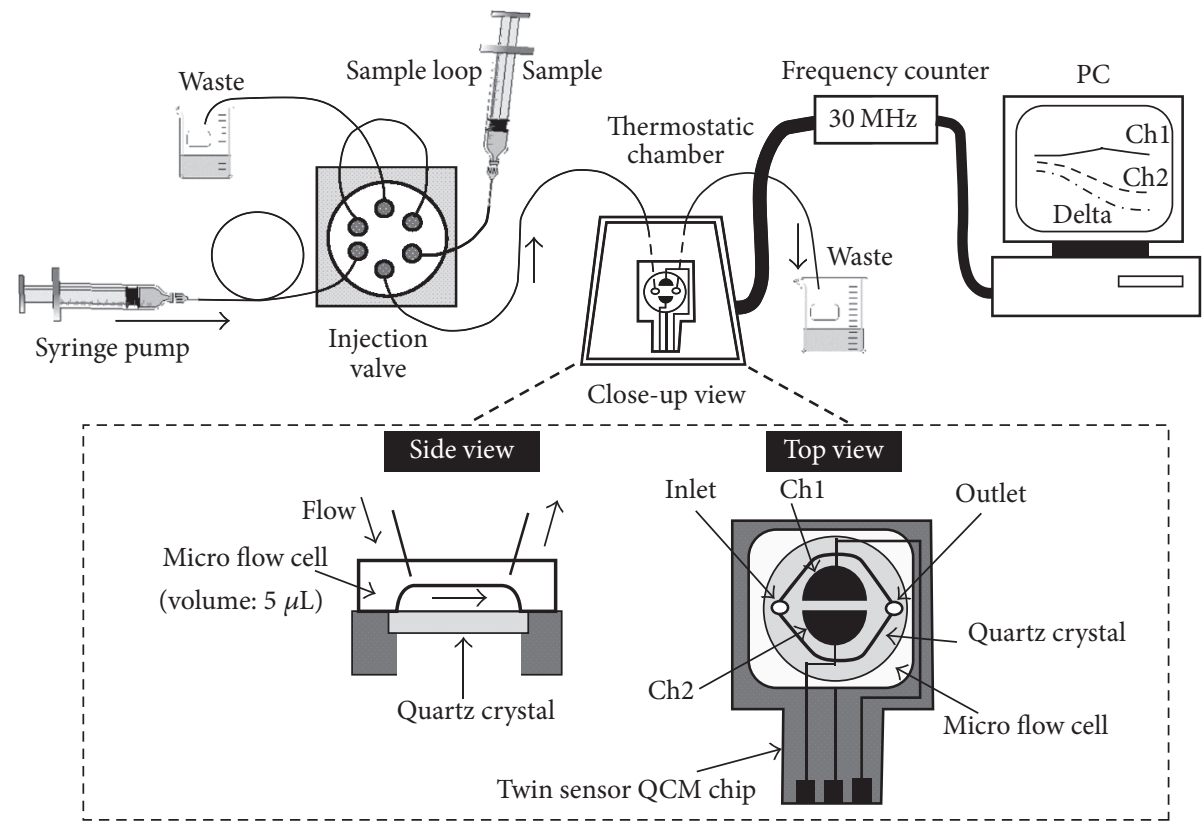

FIGURE 1: Schematic figure of our sensing system and the twin sensor chip on the QCM method.

QCM sensor can be determined using the following equation suggested by Sauerbrey [21]:

$$
\Delta m=-\Delta F \times \frac{S \times \sqrt{\rho \times \mu}}{2 N F_{0}^{2}}
$$

where $\Delta m$ is the mass change corresponding to adsorption on the active electrode surface. $\Delta F$ is the change in the oscillation frequency of a quartz crystal. $S, \rho, \mu, N$, and $F_{0}$ are the surface area of the electrode, the density of the quartz $\left(2.65 \mathrm{~g} / \mathrm{cm}^{3}\right)$, the quartz shear modulus $\left(2.95 \times 10^{11} \mathrm{~g} / \mathrm{cm} \cdot \mathrm{sec}^{2}\right)$, the order of the overtone ( $N=1$ in this case), and oscillation frequency $(30.8 \mathrm{MHz})$, respectively. Using the equation, theoretical mass change of the system was estimated to be $35 \mathrm{pg}$ of analyte with the frequency shift of $1 \mathrm{~Hz}$. Our QCM system used twin sensor and the delta between the frequency shifts on Chl and $\mathrm{Ch} 2$ was monitored. The delta remained lower than $1 \mathrm{~Hz}$ for 7 minutes, indicating that the sensing system achieved low noise level.

\subsection{Delta Measurement of Milk Sample Corresponding to Sur-} face Treatment. The surface of the sensor was very important to reduce the nonspecific adsorption of foreign substances in the milk. When the nonspecific adsorption occurs, it is acceptable that frequency shift of each channel performed similarly because of the delta measurement. Table 1 shows contact angle and the frequency difference between Chl and Ch2 (delta $=$ Ch2 - Ch1) on seven surface coating conditions after a sample injection. Here, the sample contained 10 vol\% of plain milk dissolved in PBS. Contact angle was measured using PX-G (Fibro System AB, Sweden) and that of bare Au was $83.2^{\circ}$. After the coating, contact angle degreased drastically but there were no characteristic properties corresponding to coating reagents. Bovine serum albumin (BSA) was known as a famous blocking material. We used IAS, which included BSA, at the beginning of the study. However, IAS did not reduce the nonspecific adsorption. Frequency change of each channel reached over $600 \mathrm{~Hz}$, and delta was about $100 \mathrm{~Hz}$ after the sample injection. Milk contains casein as main protein, which accounts for nearly $80 \%$ of the milk protein. So, casein might adsorb on the sensor surface mainly. Casein contents of (c), (e), (f), and (g) were approximately the same. However, coating casein was not enough to reduce the nonspecific adsorption. Frequency change of each channel was about $100 \mathrm{~Hz}$. Delta decreased to be $-10 \mathrm{~Hz}$ but more effective coating was required. Blocking reagent of N102 consists of artificial macromolecule and does not include any proteins of animal origin. Only N102 coating did not show satisfying properties of the blocking for the sample including plain milk. Delta was about $-44 \mathrm{~Hz}$. Finally, we found the surface coating method suited for the sample. In fact, N102 was coated firstly, and the blocking buffer including casein was coated secondly. Using the above condition (e, f, g), delta was recorded to be lower than $4 \mathrm{~Hz}$. For example, frequency shift corresponding to sample injection on the condition of (e) was shown in Figure 2. After the injection, frequency shift of each channel decreased because of nonspecific adsorption and viscosity changed corresponding to the sample. The frequency shift gradually increased and stayed at $-55 \mathrm{~Hz}$ after the sample solution was replaced with the buffer. However, the delta showed about $2 \mathrm{~Hz}$ at the same time. The results indicated that the sensing system using the delta did not require any offset for cortisol detection in the milk.

Surface morphology was monitored by using AFM (SPI3800, SII NanoTechnology, Japan). Figure 3 shows AFM images of prepared sensor surface scanned over $2 \mu \mathrm{m} \times 2 \mu \mathrm{m}$ with the tapping mode. Au surface on the sensor had a flat surface with $\mathrm{Ra}$ (average roughness) of $2.53 \mathrm{~nm}$. After coating 


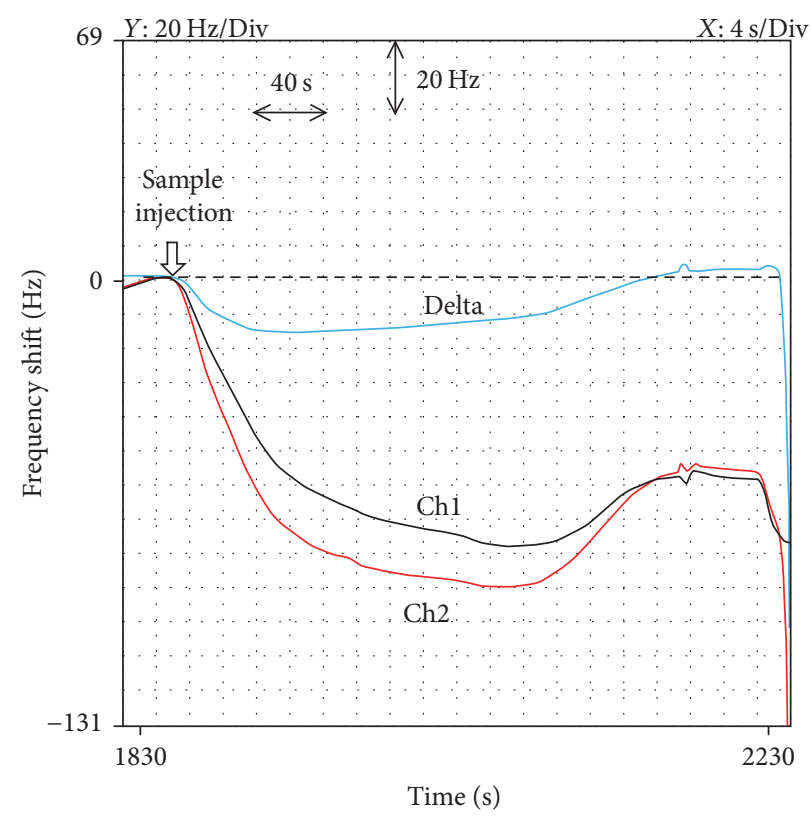

FIGURE 2: QCM responses on the sample injection. The sample contained $10 \mathrm{vol} \%$ of plain milk dissolved in PBS. The surface treatment of (e) in Table 1 was applied.

anti-Crt, surface morphology changed drastically. Antibody covered the Au surface as a whole. Surface morphology changed slightly corresponding to coating steps. Ra of prepared sensor was $2.76 \mathrm{~nm}$ after coating each reagent, which was almost the same as bare Au. The result indicated that the coating layer was very thin.

3.3. Sequential Analysis of the Sample. Flow injection analysis is suitable to examine a lot of samples. Sequential analysis system is desired for laboratory use. Uncoupling of the antibodyantigen interaction, called regeneration, was required for sequential measurements. Competitive assay was applied in this study, since the molecular weight of cortisol was 362, and the value was very small for the direct detection based on the QCM method. Sample solution including cortisol was injected with a constant concentration of tracer, which was a cortisol modified with a heavy substance. We used cortisol coupled with BSA (Crt-BSA) as the tracer because the anti-Crt had cross reactivity to the Crt-BSA. Molecular weight of BSA is $66 \mathrm{kDa}$, which is sufficiently large compared to that of cortisol. When the cortisol level was low, large frequency shift was observed, since the tracer bound to the anti-Crt mainly. On the other hand, when the cortisol level in the injected solution was higher, frequency shift was smaller, since a small amount of the tracer bound to the anti-Crt. Figure 4 shows an example of sample injection and regeneration process. The sample solution contained $10 \mathrm{vol} \%$ of plain milk and $2.5 \mu \mathrm{g} / \mathrm{mL}$ of the tracer. The frequency shif $\mathrm{t}$ on Ch1 shifted downward corresponding to the sample injection. This characteristic was due to the change of the viscosity of the sample solution. The frequency shift on Ch1 increased due to replacing the sample solution with the buffer.
By contrast, the frequency shift on Ch2 decreased drastically. After reaching the lowest point, the frequency shift gradually increased until the sample solution was replaced with the buffer the same as Ch1. As a result, the delta stayed constant after the sample solution was completely replaced with the buffer. After the injection of $4 \mathrm{mM}$ glycine- $\mathrm{NaOH}$ solution, the flow rate was changed to be $20 \mu \mathrm{L} / \mathrm{min}$ for 3 minutes for the regeneration. Then, the flow rate was set to be $6 \mu \mathrm{L} / \mathrm{min}$ again; the sensor response was reversed, since Crt-BSA deviated from anti-Crt. One determination cycle was within 10 minutes using our sensing system. As a result, we could detect the tracer without the influence of nonspecific adsorption and regenerate the QCM sensor a number of times.

To get a calibration curve, the sample solution was prepared as follows: the solution contained $10 \mathrm{vol} \%$ of plain milk and $2.5 \mu \mathrm{g} / \mathrm{mL}$ of the tracer with known concentration of cortisol. Calibration curve was obtained as shown in Figure 5. Error bars show the maximum and minimum values on each concentration $(N=4)$. The delta decreased with increasing the cortisol concentration from 0.1 to $100 \mathrm{pg} / \mathrm{mL}$. It is noted that we did not consider the cortisol level included in the plain milk. The results show that our sensing system could determine the cortisol level of the milk, which was lower than $1 \mathrm{ng} / \mathrm{mL}$.

\section{Conclusions}

We demonstrated a sequential detection method for a low molecular weight stress marker, cortisol in the milk, by using twin sensor QCM. Anti-Crt was coated on one channel (Ch2), and the blocking reagents were coated on both channels (Ch1 and Ch2) to reduce nonspecific adsorption. Ch1 was used as a reference. When the sensor surface was 

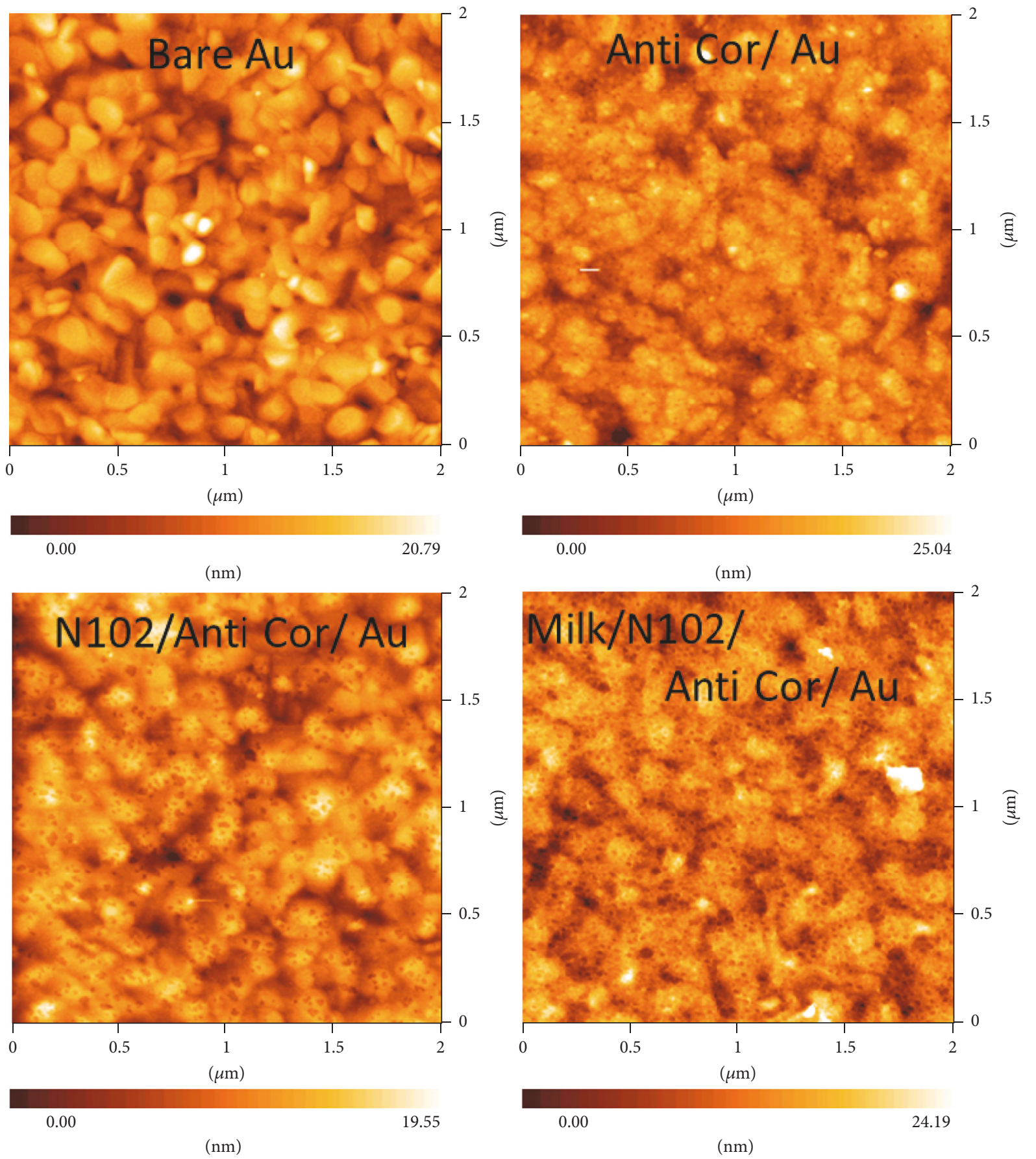

Figure 3: Surface morphology of some surface preparation by using tapping mode AFM. (a) Bare Au electrode. (b) Anti-Crt was coated on the Au electrode. (c) N102 was coated after the condition of (b). (d) The solution including 10 vol\% of plain milk was coated after the condition of (c). Scan area was $2 \mu \mathrm{m} \times 2 \mu \mathrm{m}$.

optimized, the delta (Ch2 - Ch1) showed the real bonding amount without the nonspecific adsorption and environmental influences. It took 10 minutes for one determination cycle including regeneration of antigen-antibody interaction at the flow rate of $6 \mu \mathrm{L} / \mathrm{min}$. The delta decreased with cortisol concentration from 0.1 to $100 \mathrm{pg} / \mathrm{mL}$. These results indicated that our system using the twin sensor QCM showed high sensitivity and high throughput with repeatability.

\section{Conflicts of Interest}

The authors declare that they have no conflicts of interest. 


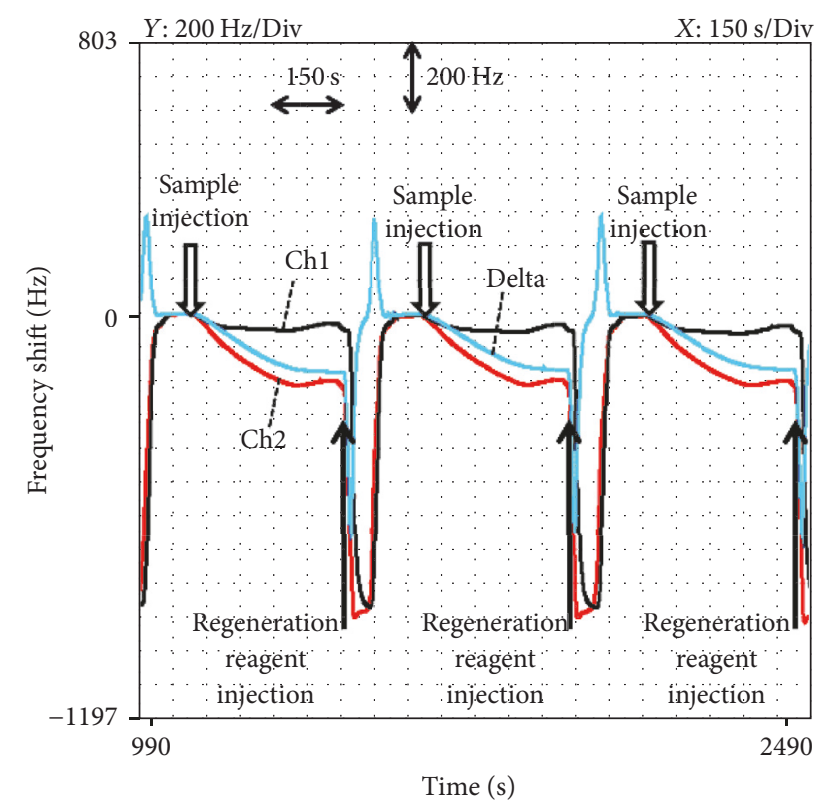

FIGURE 4: QCM responses on the sample injection and regeneration. The sample contained $10 \mathrm{vol} \%$ of plain milk and $2.5 \mu \mathrm{g} / \mathrm{mL}$ of the tracer (BSA coupled with cortisol) in PBS. $4 \mathrm{mM}$ glycine- $\mathrm{NaOH}$ solution was used for the regeneration. The surface treatment of (e) in Table 1 was applied.

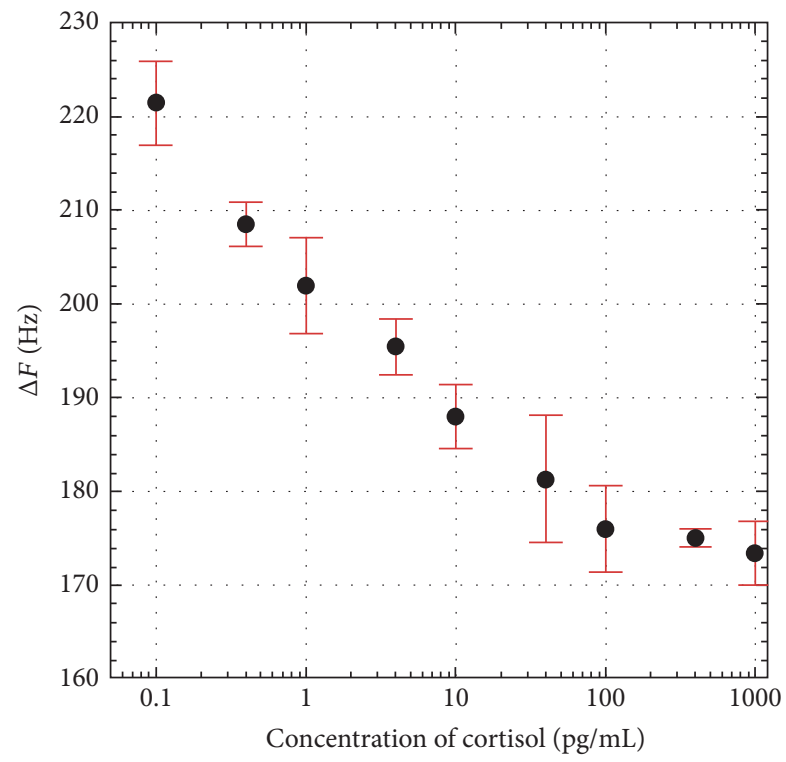

Figure 5: Calibration curve of cortisol on the sequential measurements. The condition of the sequential measurements was the same as that in Figure 4.

\section{References}

[1] M. Nagasawa, K. Mogi, and T. Kikusui, "Continued distress among abandoned dogs in fukushima," Scientific Reports, vol. 2, article 724, 2012.

[2] M. R. Kessler and D. C. Turner, "Stress and adaptation of cats (Felis Silvestris Catus) housed singly, in pairs and in groups in boarding catteries," Animal Welfare, vol. 6, no. 3, pp. 243-254, 1997.
[3] R. G. Beilharz and K. Zeeb, "Applied ethology and animal welfare," Applied Animal Ethology, vol. 7, no. 1, pp. 3-10, 1981.

[4] M. W. Fox, "Animal welfare and the dairy industry," Journal of Dairy Science, vol. 66, no. 10, pp. 2221-2225, 1983.

[5] J. L. Albright, "Status of Animal Welfare Awareness of Producers and Direction of Animal Welfare Research in the Future," Journal of dairy science, vol. 66, no. 10, pp. 2208-2220, 1983.

[6] B. A. Mallard, L. C. Wagter, M. J. Ireland, and J. C. M. Dekkers, "Effects of growth hormone, insulin-like growth factor-I, and cortisol on periparturient antibody response profiles of dairy cattle," Veterinary Immunology and Immunopathology, vol. 60, no. 1-2, pp. 61-76, 1997.

[7] F. Itoh, T. Komatsu, S. Kushibiki, and K. Hodate, "Effects of ghrelin injection on plasma concentrations of glucose, pancreatic hormones and cortisol in Holstein dairy cattle," Comparative Biochemistry and Physiology - A Molecular and Integrative Physiology, vol. 143, no. 1, pp. 97-102, 2006.

[8] Y. Saco, M. Fina, M. Giménez, R. Pato, J. Piedrafita, and A. Bassols, "Evaluation of serum cortisol, metabolic parameters, acute phase proteins and faecal corticosterone as indicators of stress in cows," Veterinary Journal, vol. 177, no. 3, pp. 439-441, 2008.

[9] D. J. Bristoe and D. S. Holmes, "Cortisol levels and anxietyrelated behaviors in cattle," Physiology \& Behavior, vol. 90, no. 4, pp. 626-628, 2007.

[10] W. R. Butler and C. K. Des Bordes, "Radioimmunoassay technique for measuring cortisol in milk," Journal of Dairy Sience, vol. 63, no. 3, pp. 474-477, 1980.

[11] S. B. Termeulen, W. R. Butler, and R. P. Natzke, "Rapidity of cortisol transfer between blood and milk following adrenocorticotropin injection," Journal of Dairy Sience, vol. 54, no. 11, pp. 2197-2200, 1981.

[12] C. Wenzel, S. Schönreiter-Fischer, and J. Unshelm, "Studies on step-kick behavior and stress of cows during milking in an automatic milking system," Livestock Production Science, vol. 83, no. 2-3, pp. 237-246, 2003.

[13] C. C. Cingi, D. F. Baser, Y. S. Karafakioglu, and A. F. Fidan, "Stress response in dairy cows related to rectal examination," Acta Scientiae Veterinariae, vol. 40, no. 3, article 1053, 2012.

[14] S. T. Long, N. C. Thinh, M. Yusuf, and T. Nakao, "Plasma Cortisol Concentrations after CIDR Insertion in Beef Cows," Reproduction in Domestic Animals, vol. 46, no. 1, pp. 181-184, 2011.

[15] M. Fukasawa, H. Tsukada, T. Kosako, and A. Yamada, "Effect of lactation stage, season and parity on milk cortisol concentration in Holstein cows," Livestock Science, vol. 113, no. 2-3, pp. 280284, 2008.

[16] T. Ito, N. Aoki, S. Kaneko, and K. Suzuki, "Highly sensitive and rapid sequential cortisol detection using twin sensor QCM," Analytical Methods, vol. 6, no. 18, pp. 7469-7474, 2014.

[17] S. Kurosawa, JW. Park, H. Aizawa, S. Wakida, H. Tao, and K. Ishikawa, "Quartz crystal microbalance immunosensors for environmental monitoring," Biosensors and Bioelectronics, vol. 22, no. 4, pp. 473-481, 2006.

[18] L. Arce, M. Zougagh, C. Arce, A. Moreno, A. Ríos, and M. Valcárcel, "Self-assembled monolayer-based piezoelectric flow immunosensor for the determination of canine immunoglobulin," Biosensors and Bioelectronics, vol. 22, no. 12, pp. 3217-3223, 2007. 
[19] C. March, J. J. Manclús, Y. Jiménez, A. Arnau, and A. Montoya, "A piezoelectric immunosensor for the determination of pesticide residues and metabolites in fruit juices," Talanta, vol. 78, no. 3, pp. 827-833, 2009.

[20] N. Mizutani, S. Korposh, R. Selyanchyn, S. Wakamatsu, and S.W. Lee, "Application of a quartz crystal microbalance (QCM) twin sensor for selective label-free immunoassay to simultaneous antigen-antibody reactions," Sensors and Transducers, vol. 137, no. 2, pp. 1-9, 2012.

[21] G. Sauerbrey, "Verwendung von Schwingquarzen zur Wägung dünner Schichten und zur Mikrowägung," Zeitschrift für Physik, vol. 155, no. 2, pp. 206-222, 1959. 


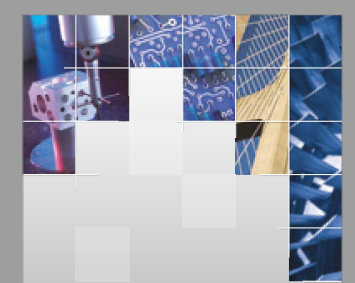

\section{Enfincering}
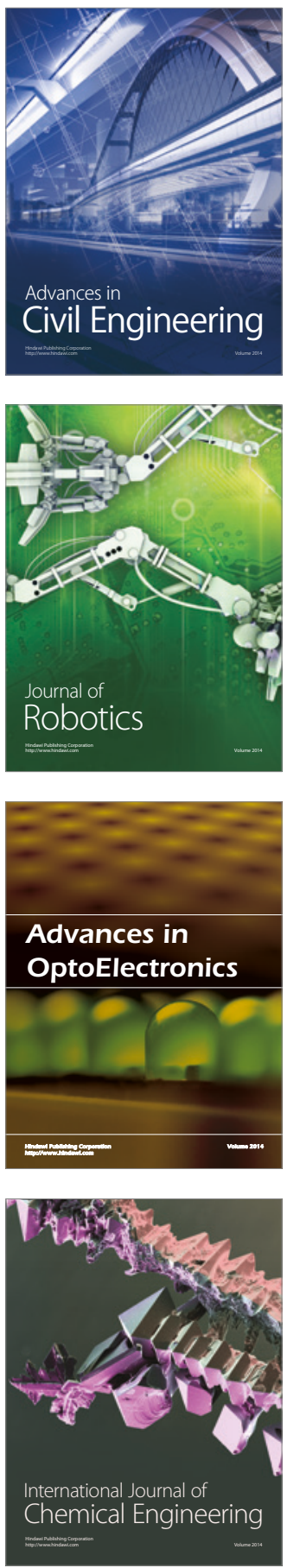

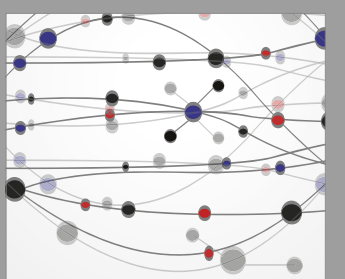

The Scientific World Journal

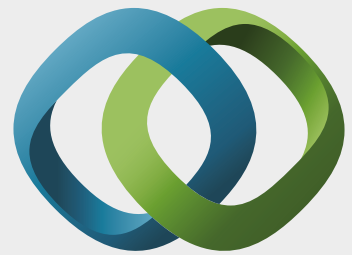

\section{Hindawi}

Submit your manuscripts at

https://www.hindawi.com
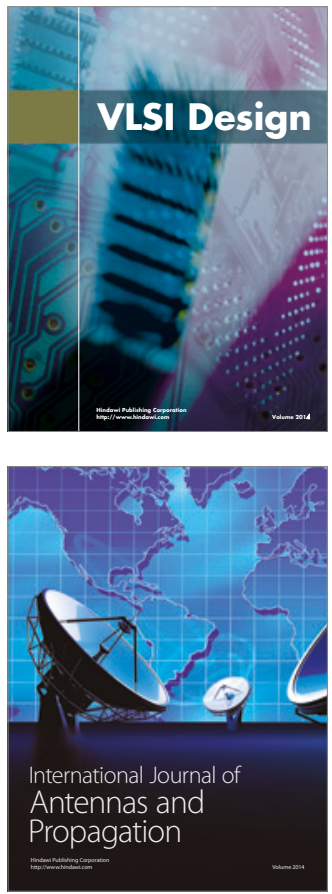

\section{Rotating}

Machinery
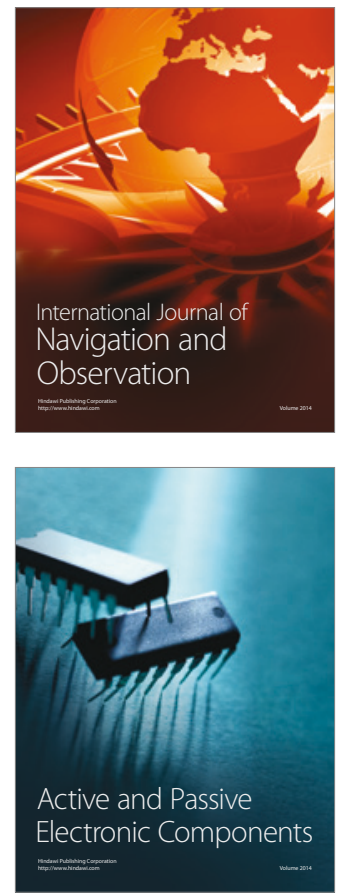
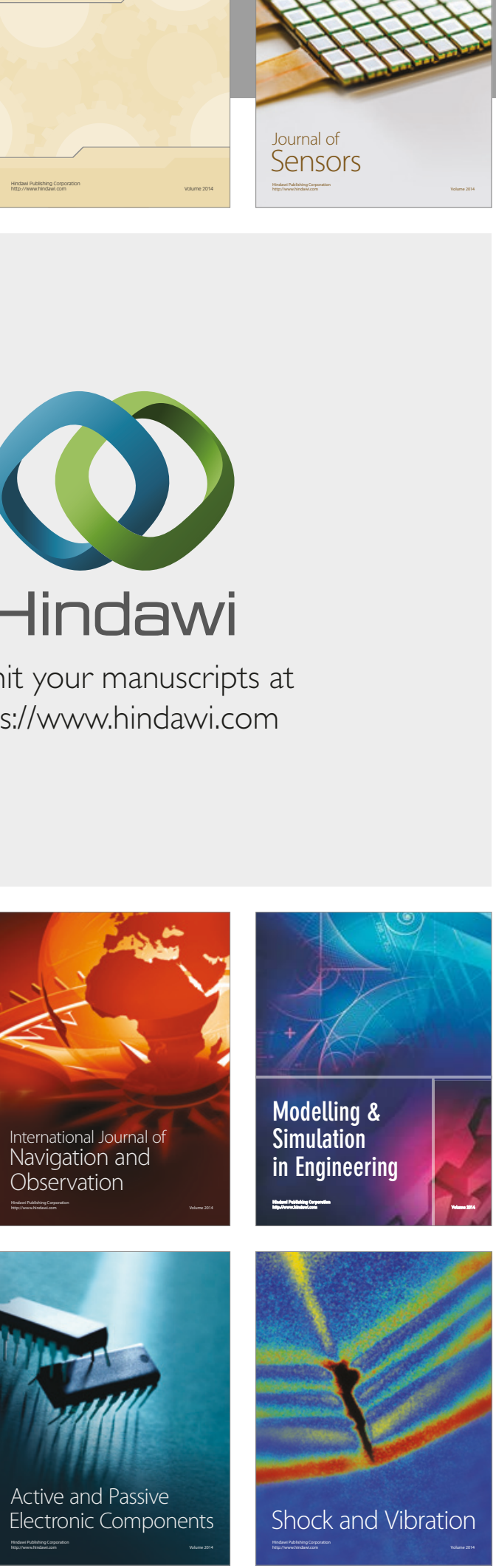
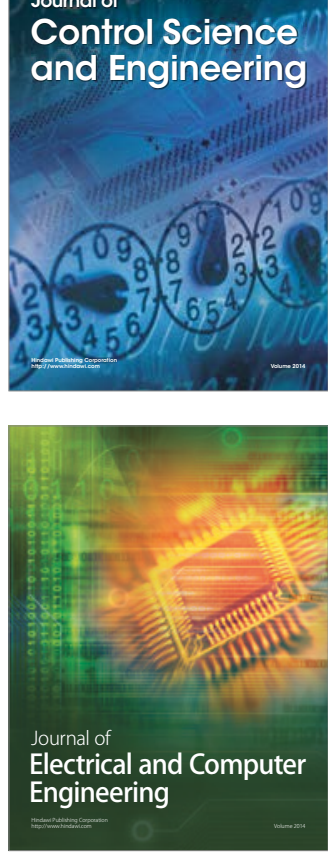

Distributed

Journal of

Control Science

and Engineering
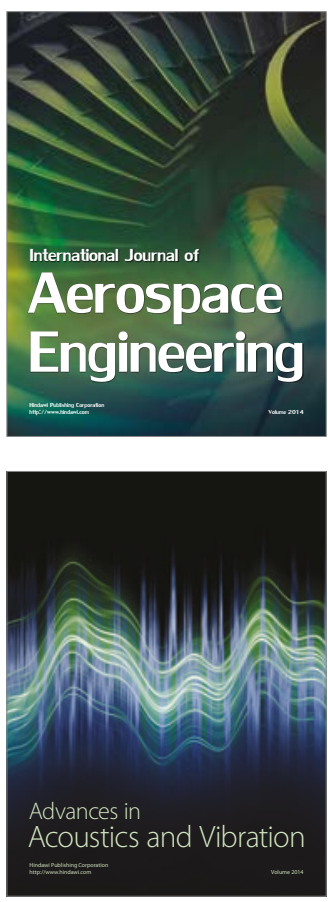

Sensor Networks 